\title{
Learning From Humans: Agent Modeling With Individual Human Behaviors
}

\section{AUTHOR(S):}

Hattori, Hiromitsu; Nakajima, Yuu; Ishida, Toru

\section{CITATION:}

Hattori, Hiromitsu ... [et al]. Learning From Humans: Agent Modeling With Individual Human Behaviors. IEEE Transactions on Systems, Man, and Cybernetics - Part A: Systems and Humans 2011, 41(1): 1-9

\section{ISSUE DATE:}

2011-01

URL:

http://hdl.handle.net/2433/131936

\section{RIGHT:}

c) 2010 IEEE. Personal use of this material is permitted. Permission from IEEE must be obtained for all other users, including reprinting/ republishing this material for advertising or promotional purposes, creating new collective works for resale or redistribution to servers or lists, or reuse of any copyrighted components of this work in other works. 


\title{
Learning From Humans: Agent Modeling With Individual Human Behaviors
}

\author{
Hiromitsu Hattori, Yuu Nakajima, and Toru Ishida, Fellow, IEEE
}

\begin{abstract}
Multiagent-based simulation (MABS) is a very active interdisciplinary area bridging multiagent research and social science. The key technology to conduct truly useful MABS is agent modeling for reproducing realistic behaviors. In order to make agent models realistic, it seems natural to learn from human behavior in the real world. The challenge presented in this paper is to obtain an individual behavior model by using participatory modeling in the traffic domain. We show a methodology that can elicit prior knowledge for explaining human driving behavior in specific environments, and then construct a driving behavior model based on the set of prior knowledge. In the real world, human drivers often perform unintentional actions, and occasionally, they have no logical reason for their actions. In these cases, we cannot rely on prior knowledge to explain them. We are forced to construct a behavior model with an insufficient amount of knowledge to reproduce the driving behavior. To construct such individual driving behavior model, we take the approach of using knowledge from others to complement the lack of knowledge from the target. To clarify that the behavior model including prior knowledge from others offers individuality in driving behavior, we experimentally confirm that the driving behaviors reproduced by the hybrid model correlate reasonably well with human behavior.
\end{abstract}

Index Terms-Modeling methodology, multiagent simulation, participatory modeling, traffic simulation.

\section{INTRODUCTION}

$\mathbf{M}$ ANY studies on multiagent-based simulation (MABS) have been done in various fields [1]-[4]. MABS yields multiagent societies that well reproduce human societies, and therefore, it is seen as an excellent tool for analyzing the real world. The key technology to implement MABS is agent modeling. This is because collective phenomena emerge from the local behaviors of many agents, i.e., the simulation result depends on each agent's microlevel behavior. Most existing studies, however, use simple or abstract agent models [5]-[7]. In order to achieve realistic agent models, it seems natural to learn from human behavior in the real world. Our research focus is to develop a methodology for generating agent models from human behavior.

Manuscript received May 29, 2009; accepted January 13, 2010. Date of publication August 12, 2010; date of current version November 10, 2010. This paper was supported in part by the Kyoto University Global COE Program: Informatics Education and Research Center for Knowledge-Circulating Society and in part by the Grant-in-Aid for Young Scientists (B) (21700161, 2009-2011) from the Japan Society for the Promotion of Science. This paper was recommended by Editor W. Pedrycz.

The authors are with the Department of Social Informatics, Graduate School of Informatics, Kyoto University, Kyoto 606-8501, Japan (e-mail: hatto@i.kyoto-u.ac.jp; nkjm@i.kyoto-u.ac.jp; ishida@i.kyoto-u.ac.jp).

Color versions of one or more of the figures in this paper are available online at http://ieeexplore.ieee.org.

Digital Object Identifier 10.1109/TSMCA.2010.2055152
Participatory modeling is a promising technology to obtain individual behavior models based on actual human behavior [8], [9]. Participatory modeling allows us to elicit a human's behavior and the reason for the behavior in particular application domains. Such information can be used as prior knowledge to explain a human's individual behavior. For a sequence of human behaviors, we can construct an individual behavior model formed on a set of prior knowledge, each piece of which can explain one of the local behaviors in the sequence.

The challenge tackled in this paper is to use participatory modeling to obtain a humanlike behavior model in the traffic domain. A human driver controls his/her vehicle based on his/her driving style. We want to construct a driver agent model that can reproduce diverse driving styles. Trying to achieve that with participatory modeling technology raises difficulties when trying to explain some driving sequences. In the real world, a human driver occasionally performs unintentional actions (i.e., actions with no logical reason). Additionally, there are cases where the driver cannot remember the reason for his/her actions. As a result, we cannot obtain sufficient prior knowledge to explain his/her driving behavior.

To permit a driver agent model to be created, even though the knowledge is insufficient, we take the approach of using complementary prior knowledge from other drivers. That is to say, if it is impossible to explain a driver's behavior using only the knowledge elicited from the driver, the knowledge acquired from other drivers is used to provide the most reasonable explanation. This approach allows us to acquire a driving behavior model that is fleshed out (patched) by knowledge from others. In order to know whether individuality in the driver's behavior is effectively preserved by the patched behavior model or not, we conduct an experiment on a driving behavior model to confirm that it well reproduces individuality in driving behavior.

In Section II, we start with some existing studies on agent modeling and then describe our participatory driver agent modeling methodology. In Section III, we show how the proposed methodology works and what behavior models can be constructed. Section IV introduces an investigation of the quality of the acquired models based on quantitative metrics. Finally, concluding remarks are given in Section V.

\section{Driver Agent Modeling}

\section{A. Current Technologies and Limitations}

In the multiagent research area, many researchers have focused on multiagent-based traffic simulations. To date, however, agent modeling with the goal of reproducing human driving behavior has not been the target of most previous works. 
Balmer et al. [10], for example, constructed a multiagent traffic simulator where each agent iteratively revises his/her preferences on the route to be traveled. In this paper, the agent model is considerably simplified since only route setting decisions are made. Halle and Chaib-draa [11] proposed an agent architecture for realizing collaborative driving by a convoy of cars. Their work, however, did not consider individuality in driving style. In contrast, Paruchuri et al. [12] tried to reproduce a variety of driving styles. However, they did not consider the realization of humanlike driving but simply introduced three driving styles defined based on three fine-tuning parameters.

Participatory technology has been used for MABSs. Sempé et al. [13] proposed how to acquire information that could explain a subject's behavior through dialogue with the subject's own agent during simulations. Unlike our work, they did not show how to identify a subject's specific behavior or how to construct behavior models. Guyot et al. [14] aimed to design interaction models by observing the emergence of power relations and coalitions during participatory simulations. Their research goal is different from ours, which focuses on the agents' internal mechanism.

Reinforcement learning (RL) seems a promising technology for obtaining driving behavior models [15], [16]. By agent modeling with RL technologies, we may be able to obtain a computational model of drivers, but the acquired models are limited to simply rerunning human driving logs. Their approach does not yield individuality in driving style.

\section{B. Participatory Driver Agent Modeling}

1) Outline: During participatory driver agent modeling, we construct driving behavior models from human driving data by collaborating with the human subjects. Using the participatory modeling allows us to construct behavior models from not only our (modeler's) knowledge but also the actual behavior of the human subjects. The modeling process consists of the following five steps.

1) Collect human driving log data from trials performed on a 3-D virtual driving simulator.

2) Together with domain experts, identify individual driving behaviors by the investigation of collected log data.

3) Collect prior knowledge that can form a driving behavior model by interviewing the subjects of the driving simulation.

4) Select meaningful prior knowledge and formally express it.

5) Construct a driving behavior model that can explain the human subject's actions based on hypothetical reasoning [17].

We detail each step in the remainder of this section.

2) Collecting Driving Log on 3-D Virtual Driving Simulator: In order to construct a driving behavior model, we need realistic driving data from humans. In the real world, however, it is hard to collect sufficient driving data in actual traffic environments due to the difficulties of setting up the experimental environment. Thus, we use a 3-D virtual driving simulator that has a lifelike cockpit and a wide screen that can display a virtual

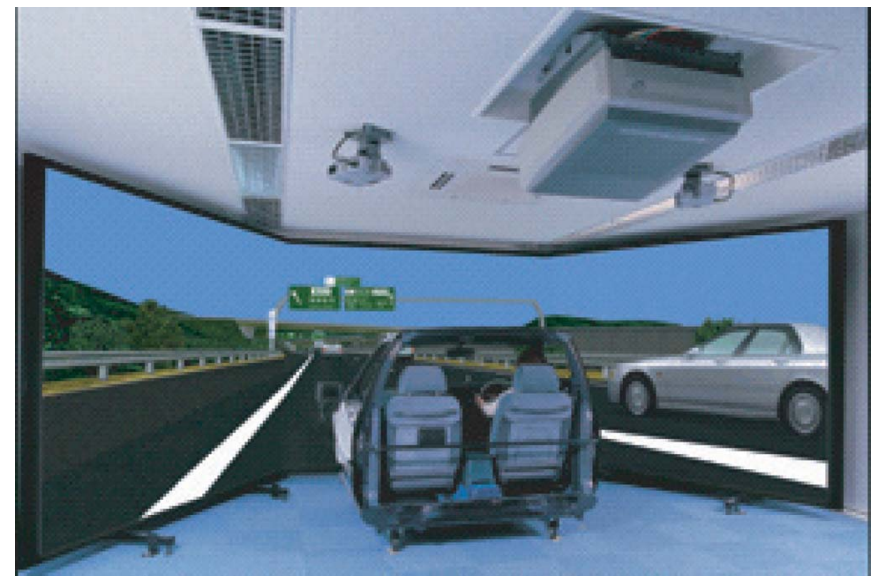

Fig. 1. Three-dimensional virtual driving simulator used for collecting driving $\log$ data.

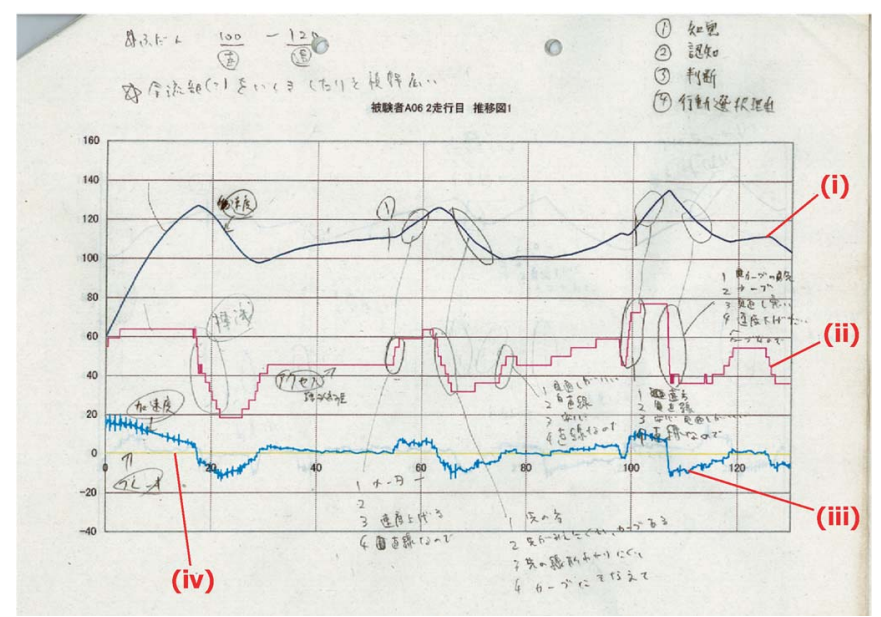

Fig. 2. Example of a chart made from driving log data. Graphs (i), (ii), (iii), and (iv) denote the speed, acceleration, usage of accelerator, and usage of brake, respectively. The circles on the graph represent the subject's specific behaviors identified by traffic engineers.

environment (see Fig. 1). ${ }^{1}$ Such simulations are often used to train drivers, and so, our simulator is expected to yield realistic driving data. Fig. 2 is one example of a chart made from driving $\log$ data. As shown, we can get information on transitions in running speed (the graph at the top), acceleration (graph second from the top), and the usage of accelerator/brake (graphs at the bottom).

3) Identifying Individual Behaviors With Domain Expert: We investigated the collected driving log data to identify each subject's individual driving behavior. For the investigation, we use the following data collected for each subject:

1) mileage (in kilometers) - the road distance from the origin;

2) speed (in kilometers per hour)-the speed of the subject's car;

3) acceleration (in meters per second) - the acceleration of the subject's car;

\footnotetext{
${ }^{1}$ This virtual driving simulator is located in the Division of Global Architecture, Graduate School of Engineering, Osaka University, Suita, Japan.
} 
TABLE I

Predicates to Represent ACtions

\begin{tabular}{|c||l|}
\hline \multicolumn{1}{|c||}{ Predicate } & Description \\
\hline Straight $(\mathrm{X})$ & $\mathrm{X}$ is a straight road. \\
\hline Curve $(\mathrm{X})$ & $\mathrm{X}$ is a curve. \\
\hline Uphill $(\mathrm{X})$ & $\mathrm{X}$ is an uphill. \\
\hline Downhill $(\mathrm{X})$ & $\mathrm{X}$ is a downhill. \\
\hline On $(\mathrm{X}, \mathrm{Y})$ & $\mathrm{Y}$ is driving on $\mathrm{X}$. \\
\hline InSight $(\mathrm{X}, \mathrm{Y})$ & Y can see $\mathrm{X}$. \\
\hline OverDesiredSpeed $(\mathrm{X})$ & The speed of a vehicle $\mathrm{X}$ exceeds the desired speed. \\
\hline UnderDesiredSpeed $(\mathrm{X})$ & The speed of a vehicle $\mathrm{X}$ is under the desired speed. \\
\hline OverCurveSpeed $(\mathrm{X}, \mathrm{Y})$ & The speed of a vehicle $\mathrm{Y}$ is too high in a curve $\mathrm{X}$. \\
\hline SpeedUp $(\mathrm{X})$ & A vehicle $\mathrm{X}$ is speeding up. \\
\hline SlowDown $(\mathrm{X})$ & A vehicle $\mathrm{X}$ is slowing down. \\
\hline Accelerate $(\mathrm{X})$ & A vehicle $\mathrm{X}$ is accelerating. \\
\hline Decelerate $(\mathrm{X})$ & A vehicle $\mathrm{X}$ is decelerating. \\
\hline
\end{tabular}

4) usage of acceleration (in percent) - the usage of accelerator, i.e., accelerator pedal position. ${ }^{2}$

We try to capture an individual's behavior by investigating his/her driving log data. In particular, the speed/acceleration transitions provide a lot of useful data. The experiment shown in Section III confirms that different drivers have different driving styles, even in identical conditions. Therefore, the sequence of each local driving behavior can be taken as an expression of individuality in driver's behavior. Fig. 2 shows some transitions on graph (ii) in the figure (marked by circles); they represent the results of specific operations. Since it was difficult for us to accurately identify key transitions from the $\log$ data, we elicited the help of domain experts (i.e., traffic engineers).

4) Interview of Subjects: We interviewed the subjects after they participated in the driving simulation. The purpose of the interview was to gather information on their specific operations, identified in the previous step, for generating prior knowledge. We use screenshots of the simulation and charts like Fig. 2 in order to make it easy for the subjects to remember the reasons for his/her actions in the simulation.

In the interview, we asked each subject about the following four points for each specific operation:

1) reason/motivation for the operation: confirmation of the reason or motivation for the operation;

2) target of the subject's gaze:

confirming what the subject was really gazing at;

3) recognized target:

confirming what the subject recognized;

4) evaluation of the recognition:

confirming how the subject evaluated the result of the recognition.

Fig. 2 shows some notes on several of the transitions. For example, the notes at the center of the figure show the following responses.

1) Getting ready for a curve.

2) The road in front of me.

${ }^{2}$ In this paper, the rate is $0 \%$ when the pedal is not depressed, while the rate is $100 \%$ when the pedal is fully depressed.
3) The curve is close, and I cannot see into the curve.

4) The road forward is unclear.

Our analyses of the interview log and charts yielded information on the subjects' operations under a range of conditions, i.e., "sense-act" information. We use such information as prior knowledge and represent it as driving rules, each of which denotes a driving operation made under a certain condition.

5) Formal Representation of Collected Knowledge: We first cleaned up the collected prior knowledge (i.e., driving rules). For example, in the real example shown in Section III, we obtained knowledge such as "If I feel fine, I'll step on the accelerator." This kind of knowledge, which is related to feeling, is not suitable for modeling because we cannot observe the internal states of humans. Thus, we first eliminated such knowledge. The knowledge remaining is represented using formal expressions based on predicate logic. After a discussion with traffic engineers, we fixed some predicates to represent prior knowledge (see Table I).

These predicates were also used to formally describe the observations extracted from the driving log data. An observation describes what the subject noticed and how he/she operated his/her vehicle in the situation presented.

This formal description of prior knowledge and observations allows us to use them in the next step of model construction.

6) Construction of Driving Behavior Models:

a) Formalizing the problem: In this paper, we assume that a subject decides his/her next operation based on the surrounding environment, as observed from his/her viewpoint. We denote the environment observed by the subject as $E$; it consists of conjunctions of literals about the environment. The environment at time $t$ is tagged as $E_{t}$. The driving model $\mathcal{M}$ is a set of prioritized driving rules $\langle P, \preceq\rangle$, which is a set of driving rules, where $\preceq$ represents the priorities of each rule in $P$. $P$ is a subset of Rules, which is the set of rules obtained from all subjects. Therefore, each driving model may consist of prior knowledge obtained from several human subjects. $\preceq$ is a subset of the Cartesian product, i.e., Rules $\times$ Rules. Each driving rule in Rules is denoted as rule $e_{i}(0 \leq i \leq j \leq \mid$ Rules $\mid)$, so that $\left\langle\right.$ rule $_{i}$, rule $\left._{j}\right\rangle \in \preceq$ is described as rule $_{i} \preceq$ rule $_{j}$.

In order to apply hypothetical reasoning [17] to the modeling of driving behaviors, we define driving rules and an operation 
selection mechanism as domain knowledge $\Sigma$. An element of domain knowledge is indicated by $\sigma_{k}(0 \leq k \leq|\Sigma|)$. We hypothesize which driving rules are employed by the target subject $\left(\right.$ rule $\left._{i} \in P\right)$ and which rules take priority $\left(\right.$ rule $_{i} \preceq$ rule $\left._{j}\right)$. A set of these hypotheses is indicated by $H$. Additionally, we describe the subject's behavior from the beginning of the simulation on the 3-D simulator, namely, 0 , to the end of the simulation, namely, end, as observation $G$, and the observation at time $t$ is denoted as $G_{t}$.

The operation selection mechanism is defined as follows.

Definition 1 (Driving operation selection: $\left.\sigma_{1}\right)$ : $\left(\exists\right.$ rule $_{i}\left(\right.$ rule $_{i} \in P \wedge$ rule $_{i}=\max \prec\{$ rule $\mid$ Applicable $($ rule, $\left.\left.\left.\left.E_{t}\right)\right\}\right)\right) \Rightarrow$ Do $\left(\right.$ operation $\left(\right.$ rule $\left.\left._{i}\right)\right)$ Here, Applicable and Do are pseudopredicates. The former means that the condition part of a rule is satisfied, and the latter means that the subject initiates an operation. Function operation returns the operation initiated by the subject when he/she executes rule $_{i} . \sigma_{1}$ means that a subject employs rule $_{i}$, the rule that has the highest priority among all applicable operations at $E_{t}$.

Definition 2 (Continuation of operation: $\sigma_{2}$ ): A subject can continue his/her current operation.

Definition 3 (Constraint: $\left.\sigma_{3}\right): \forall$ rule $_{i}$, rule $_{j}\left(\right.$ rule $_{i}$, rule $_{j} \in P \wedge\left(\operatorname{condition}\left(\right.\right.$ rule $\left._{i}\right)=\operatorname{condition}\left(\right.$ rule $\left.\left._{j}\right)\right) \Rightarrow$ $\left(\right.$ operation $\left(\right.$ rule $\left._{i}\right)=$ operation $\left(\right.$ rule $\left.\left._{j}\right)\right) \quad \sigma_{3}$ means that $P$ does not include driving rules that have identical condition parts but different operations. Here, the function condition returns the precondition of its argument.

We define $G$ and $G_{t}$ in the following.

Definition 4 (Observation $G$ ): $G \equiv\left(G_{0} \wedge \ldots \wedge G_{t} \wedge \ldots \wedge\right.$ $G_{\text {end }}$ ).

Definition 5 (Observation $\left.G_{t}\right): G_{t} \equiv\left(E_{t} \Rightarrow A_{t}\right)$. $A_{t}$ is the literal represented by predicate Do.

The observations, present in the driving log data, are described using the predicates shown in Table I. We use road structure, driving speed, and acceleration pedal operation as observations. A typical description is as follows.

Example 1 (Description of observation): Curve $\left(\right.$ Curve $\left._{1}\right) \wedge$ InSight $\left(\right.$ Curve $_{1}$, self $) \wedge \operatorname{Uphill}\left(\right.$ Uphill $\left._{1}\right) \wedge \mathrm{On}\left(\right.$ Uphill $_{1}$, self) $\wedge$ OverDesiredSpeed(self) $\Rightarrow$ Do (ReleaseAccel(self)).

This observation means that the subject releases the accelerator when he/she sees Curve $_{1}$ (InSight), his/her vehicle is driving $U_{p h i l l}(\mathrm{On})$, the speed of a vehicle exceeds the desired speed (OverDesiredSpeed), and he/she is decelerating (ReleaseAccel).

b) Model acquisition process: We applied a modeling method based on hypothetical reasoning [18] to acquire a driving behavior model of each human subject. The method should yield models that can explain $G$ in association with $\Sigma$ and $H$. As mentioned previously, $\Sigma$ is the operation selection mechanism and operation rules, and $H$ indicates which driving rule is employed by the subject, i.e., which rule has priority.

The major steps of the model acquisition algorithm are as follows.

1) The driving model at time $t-1, \mathcal{M}=\langle P, \preceq\rangle$, is input.

2) If the target subject continues the same driving operation as that at time $t-1$, the algorithm just returns $\mathcal{M}$. (a)

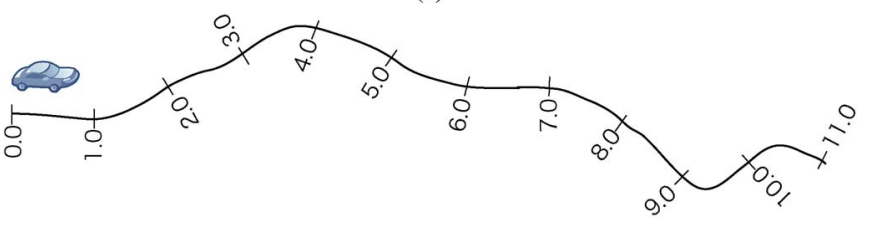

(b)

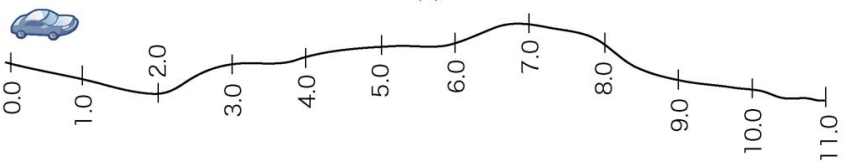

Fig. 3. Road structure in the 3-D driving simulator. (a) Horizontal alignment. (b) Vertical alignment.

3) If the subject initiates a new operation at time $t$, driving rule $p$, which is applicable to $E_{t}$ and can explain $A_{t}$, is chosen from $P . p$ is assigned higher priority than all other rules applicable to $E_{t}$ in $P$ ( $\preceq$ is updated to $\preceq^{\prime}$ ); finally, $\mathcal{M}=\left\langle P, \preceq^{\prime}\right\rangle$ is returned. The goal of the algorithm is to obtain the minimal explanation. Therefore, the algorithm first tries to find an applicable rule in the current $P$ to avoid adding another rule.

4) If there is no applicable driving rule in $P$, driving rule $p$, which is applicable to $E_{t}$, is chosen from Rules. $p$ is assigned higher priority than all other rules applicable to $E_{t}$ in Rules ( $\preceq$ is updated to $\left.\preceq^{\prime}\right)$; finally, $\mathcal{M}=(P \cup$ $\left.\{p\}, \preceq^{\prime}\right)$ is returned.

If $P \cup\{p\}$ is inconsistent, the algorithm returns "fail."

For model acquisition, explanation-based learning (EBL) [19] is another potential technique. In EBL, an observation can be explained by using domain knowledge and training data without making a hypothesis. On the contrary, in hypothetical reasoning, an observation can be explained by using domain knowledge under a hypothesis, and the hypothesis could be considered as true iff it is consistent with the domain knowledge. When we try to construct driving models, we do not know which rules are used by human subjects and which rule has priority. Thus, we are required to construct models based on hypothetical reasoning with hypothesis, such as "rule $e_{i}$ was prioritized" and "this subject employed rule $_{i}$. "

\section{Real Example of Driver Agent Modeling}

We conducted an experiment to construct driver agent models based on the modeling methodology mentioned earlier. In this section, we show how the proposed methodology works and what models were constructed in the experiment.

\section{A. Setting and Modeling Process}

First, we describe the setting of the driving simulation used to collect driving log data. In this paper, we used an $11-\mathrm{km}$ virtual highway whose layout is shown in Fig. 3. For simplicity, in this paper, each human subject drove alone, so that we could elicit prior knowledge representing just the driving operations. There were 36 subjects, and each had an experience in using the 3-D simulator. We could successfully obtain prior knowledge (i.e., 
TABLE II

ObTaIned KNOWLEDGE From Human SubJeCts

\begin{tabular}{|l||l|}
\hline \multicolumn{1}{|c||}{ RuleID } & Description of a rule \\
\hline rule $_{\mathbf{0 1}}$ & If a subject is driving a curve, he/she releases the accelerator. \\
\hline rule $_{\mathbf{0 2}}$ & If a subject is driving a straight, he/she steps on the accelerator. \\
\hline rule $_{\mathbf{0 3}}$ & If a subject is driving uphill, he/she steps on the accelerator. \\
\hline rule $_{\mathbf{0 4}}$ & If a subject is driving downhill, he/she releases the accelerator. \\
\hline rule $_{05}$ & If a subject sees a curve ahead, he/she releases the accelerator. \\
\hline rule $_{06}$ & If a subject sees a straight ahead, he/she steps the accelerator. \\
\hline rule $_{\mathbf{0 7}}$ & If a subject sees an uphill ahead, he/she steps on the accelerator. \\
\hline rule $_{08}$ & If a subject sees a downhill ahead, he/she releases the accelerator. \\
\hline rule $_{09}$ & $\begin{array}{l}\text { If the speed exceeds the desired speed, a subject releases the } \\
\text { accelerator. }\end{array}$ \\
\hline rule $_{10}$ & $\begin{array}{l}\text { If a subject is driving under the desired speed, he/she steps on the } \\
\text { accelerator. }\end{array}$ \\
\hline rule $_{11}$ & If a vehicle slow down, he/she steps on the accelerator. \\
\hline rule $_{12}$ & If a vehicle speed up, he/she release the accelerator. \\
\hline
\end{tabular}

driving rules) from all subjects through a collaboration with traffic engineers, but some subjects provided only one or two rules. The set of obtained prior knowledge is shown in Table II. Because the experiment was held on a virtual highway with no other cars, all subjects used just the accelerator. In a few cases, the subject used the brake but had no logical reason for doing so. Prior knowledge indicated how the human subject might decide to use the accelerator considering surrounding road structure, current velocity, and own desired speed.

We then formally expressed the obtained prior knowledge by using the predicates that we defined to describe observations. Example 2 shows a description of prior knowledge.

Example 2 (Description of prior knowledge):

rule $_{1}$ :

if $\operatorname{Curve}(x) \wedge \mathrm{On}(x$, self) then ReleaseAccel(self)

rule :

if $\operatorname{Curve}(x) \wedge \operatorname{InSight}(x$, self $)$ then ReleaseAccel(self)

rule $_{7}$ :

if $\operatorname{Uphill}(x) \wedge \operatorname{InSight}(x$, self)then Accelerate(self)

For instance, rule $e_{1}$ means that if there is a curve $x$ (Curve $(x))$ and the subject ("self") is driving on the curve $x(\operatorname{On}(x$, self $))$, he/she releases the accelerator (ReleaseAccel(self)). Rule 5 means that if there is an upcoming curve $x$ (Curve $(x))$ and if the subject ("self") sees curve $x$ (InSight $(x$, self $))$, he/she releases the accelerator (ReleaseAccel(self)). Rule $e_{7}$ also means that if a hill is to be climbed $x$ (Uphill $(x))$ and the subject sees that, he/she steps on the accelerator (Accelerate(self)).

Finally, we used the obtained knowledge and observations to construct driving behavior models using the algorithm shown in Section II-B6b. In the algorithm, steps 3) and 4) are the essential steps that evaluate the ability of the current model to explain the human subject's behaviors and choose appropriate rules from the set of prior knowledge. In order to make it clear how the algorithm works to construct a model, we show here a part of the modeling process to prove that Do(ReleaseAccel(self)). Note that we assume Rules to be $\left\{\right.$ rule $_{1}$, rule $_{5}$, rule $\left._{13}\right\}$, and $h_{T-2}$, the operation model acquired from $G_{0}, \ldots, G_{T-2}$, to be $\left\{\right.$ rule $\left._{13} \in P\right\}$. Here, rule $e_{13}$ is a fictional rule repre- senting "if a subject is driving a curve, he/she steps on the brake."

1) In order to derive Do(ReleaseAccel(self)), due to $\sigma_{1}$, it is required to prove that action $\left(\right.$ rule $\left._{i}\right)=$ ReleaseAccel(self), rule $_{i} \in P$, and that rule $_{i}=$ $\max _{\preceq}\left\{\right.$ rule $\mid$ Applicable $\left(\right.$ rule, $\left.\left.E_{t-1}\right)\right\}$ are true.

2) Rule $_{1}$ and rule $_{5}$ can satisfy the first condition, i.e., $\operatorname{action}\left(\right.$ rule $\left._{i}\right)=$ ReleaseAccel(self), since their consequents are Initiate(ReleaseAccel(self)).

3) Substitute rule $_{1}$ for rule $_{i}$.

a) Choose the assumption, i.e., rule $e_{1} \in P$, from $H$ to prove that rule $_{1} \in P$ is true. However, rule $e_{13}$ and rule $_{1}$ in $P$ are incompatible according to $\sigma_{3}$, and thus, we are forced into backtracking.

4) Substitute rule $_{5}$ for rule $_{i}$.

a) Choose an assumption, i.e., rule $e_{5} \in P$, from $H$ to prove that rule $_{5} \in P$ is true.

b) Choose an assumption, i.e., rule $1 \preceq$ rule $_{5}$, rule $_{13} \preceq$ rule $_{5}$ from $H$ to prove that rule $_{5}=$ $\max _{\preceq}\left\{\right.$ rule $\mid$ Applicable $\left(\right.$ rule, $\left.\left.E_{t-1}\right)\right\}$ is true.

c) Since $\quad h_{t-1}=\left\{\left\{\right.\right.$ rule $_{13}$, rule $\left._{5}\right\},\left\{\right.$ rule $_{1} \preceq$ rule $_{5}$, rule $_{13} \preceq$ rule $\left.\left._{5}\right\}\right\}$ is acquired.

This process is iterated until $G_{\text {end }}$ can be explained; the result is a driving model.

\section{B. Acquired Driving Behavior Models}

In the experiment, we could construct driving behavior models for all subjects. In this section, we show some examples of the acquired driving behavior models. Table III shows a set of driving rules and their priorities. Fig. 4 shows the transitions in running speed and acceleration of the subjects and their corresponding driver agents. In Fig. 4, the vertical and horizontal axes represent speed (in kilometers per hour) and mileage (in kilometers), respectively. The bold blue and bold green lines plot the subject's running speed and acceleration, respectively. The thin red and thin orange lines represent the driver agent's running speed and acceleration, respectively.

Case 1 for $S_{1}$ : The driving behavior model of subject $S_{1}$ consists of six driving rules and the relationships defining their 
TABLE III

EXAMPles of ACQuired DRIVING BeHAVIOR MODElS

\begin{tabular}{|c|c|}
\hline ID & Driving behavior model \\
\hline$S_{1}$ & 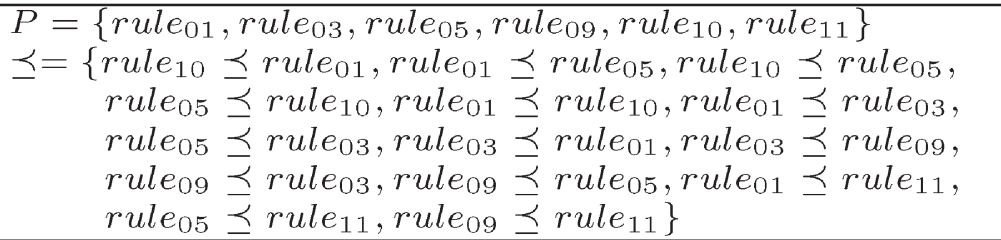 \\
\hline$S_{2}$ & 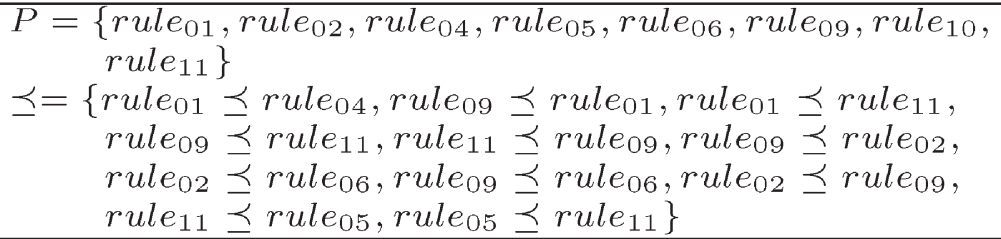 \\
\hline$S_{3}$ & 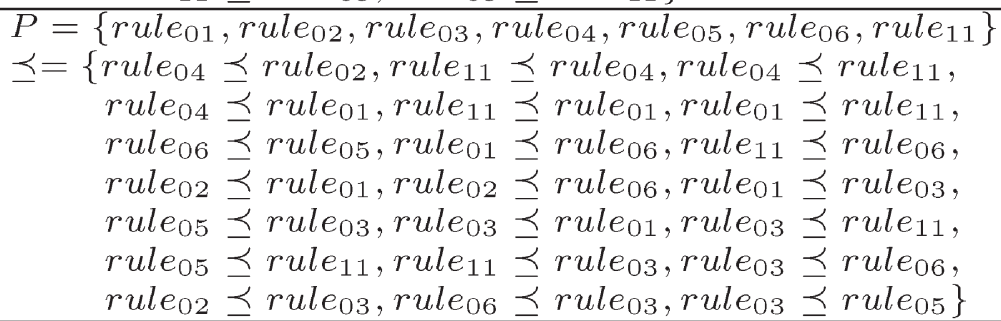 \\
\hline
\end{tabular}

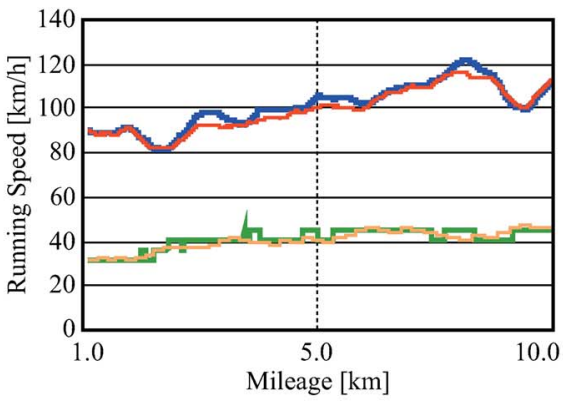

(A-1)

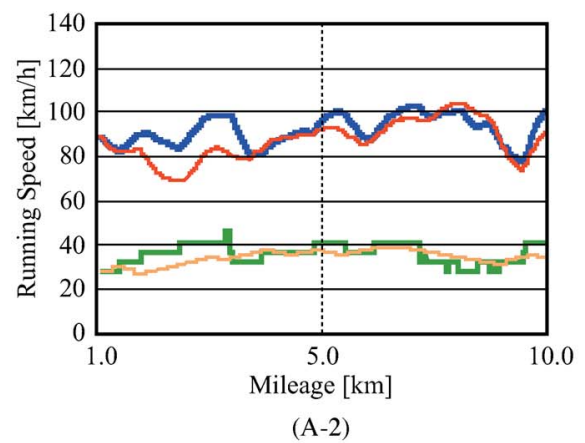

(A-2)

(a)

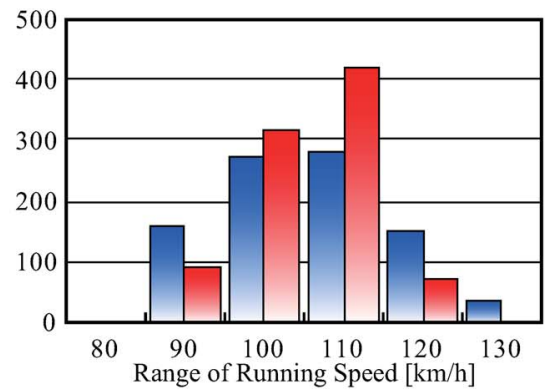

(B-1)

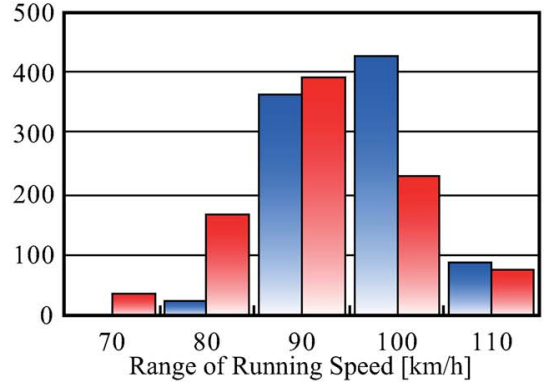

(B-2)

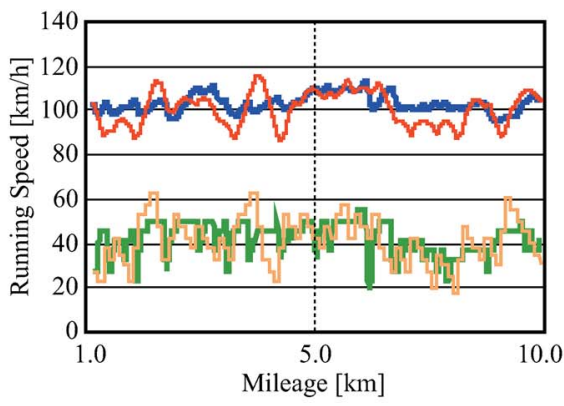

(A-2)

(b)

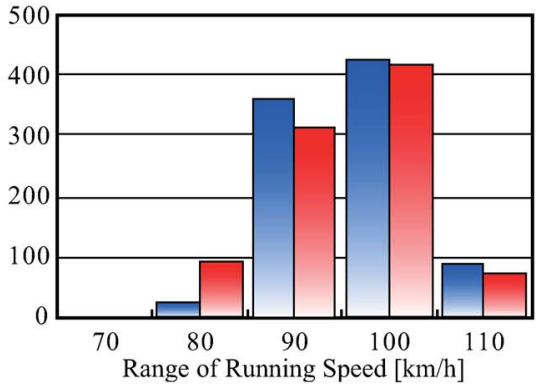

(B-2)

Fig. 4. Transitions in running speed and acceleration of human subjects and corresponding driver agent. (a) Transition of running speed and acceleration. (b) Distribution of running speed.

priorities. The road section of $1-7 \mathrm{~km}$ is a gentle ascending slope with some curves, as shown in Fig. 3. $S_{1}$ drove under his/her desired speed $(120 \mathrm{~km} / \mathrm{h})$ in this zone [see Fig. 4(A-1)]. $S_{1}$ 's behavior model can reproduce his/her driving log by the application of three rules, namely, rule $_{03}$, rule $_{07}$, and rule 10 . The running speed is increased by these rules. After the 7-km point, the road curves downhill. Because $S_{1}$ 's model does not include a rule to release the accelerator, at first, the running speed is continuously increased. However, once the speed exceeds the desired speed, rule $_{09}$ is fired, and the accelerator pedal is released. If the speed becomes too slow, this model can recover because rule $_{11}$, which is used to speed up when vehicle speed becomes too slow, is prioritized over rule $_{01}$ and rule $_{05}$, which are used to release the accelerator in a curve. 
TABLE IV

Comparison Between Humans' Log Data And Agents' Log Data. (a) Correlation Value for the Running Speed of Humans and Agents. (b) Average and Standard Deviationof the Running Speed

\begin{tabular}{|c|c||c|c|c|c|c|c|}
\hline & \multicolumn{2}{|c||}{} & \multicolumn{2}{c|}{$S_{1}$} & \multicolumn{2}{c|}{$S_{2}$} & \multicolumn{2}{c|}{$S_{3}$} \\
\hline & & Human & Agent & Human & Agent & Human & Agent \\
\hline \hline \multirow{2}{*}{$S_{1}$} & Human & 1 & $*$ & $*$ & $*$ & $*$ & $*$ \\
\cline { 2 - 8 } & Agent & $\mathbf{0 . 9 5}$ & 1 & $*$ & $*$ & $*$ & $*$ \\
\hline \multirow{2}{*}{$S_{2}$} & Human & 0.66 & 0.62 & 1 & $*$ & $*$ & $*$ \\
\cline { 2 - 8 } & Agent & 0.90 & 0.87 & $\mathbf{0 . 7 2}$ & 1 & $*$ & $*$ \\
\hline \multirow{2}{*}{$S_{3}$} & Human & 0.30 & 0.21 & 0.59 & 0.34 & 1 & $*$ \\
\cline { 2 - 8 } & Agent & 0.05 & -0.03 & 0.1 & -0.03 & $\mathbf{0 . 6 1}$ & 1 \\
\hline
\end{tabular}

(a)

\begin{tabular}{|c|c||r|r|}
\hline ID & Entity & Average & Standard Dev. \\
\hline \hline \multirow{2}{*}{$S_{1}$} & Human & 100.9 & 10.6 \\
\cline { 2 - 4 } & Agent & 95.8 & 9.1 \\
\hline \multirow{2}{*}{$S_{2}$} & Human & 91.6 & 7.18 \\
\cline { 2 - 4 } & Agent & 86.8 & 9.5 \\
\hline \multirow{2}{*}{$S_{3}$} & Human & 102.9 & 5.32 \\
\cline { 2 - 4 } & Agent & 100.2 & 8.22 \\
\hline
\end{tabular}

(b)

Case 2 for $S_{2}$ : The driving behavior model of subject $S_{2}$ includes eight driving rules. In Fig. 4(A-1) and (A-2), $S_{2}$ 's behavior looks similar to that of $S_{1}$. The difference is apparent in the 7-9-km region. $S_{2}$ drove at around $100 \mathrm{~km} / \mathrm{h}$, while $S_{1}$ exceeded $100 \mathrm{~km} / \mathrm{h} . S_{2}$ 's model can reproduce this difference in driving behavior. It includes rule $_{4}$, representing "if the subject sees a downhill ahead, he/she releases the accelerator." Therefore, $S_{2}$ 's model lowers the speed. This is one example of realizing individuality in driving style.

Case 3 for $S_{3}: S_{3}$ was a driver whose driving style was hard to explain and reproduce. The frequency of acceleration is relatively high. This is because he/she seems keen to maintain his/her desired speed exactly $(100 \mathrm{~km} / \mathrm{h})$. As shown in Fig. 4(A-3), $S_{3}$ speeds up little by little to just over $100 \mathrm{~km} / \mathrm{h}$. The model of $S_{3}$ can reproduce this driving style by including both rule $_{10}$ ("If the vehicle speeds up, he/she releases the accelerator") and rule $_{12}$ ("If the subject is driving under the desired speed, he/she steps on the accelerator"). A comparison of the transitions in acceleration makes it clear that $S_{3}$ 's model yields a behavior that is different from those of the other two models.

\section{Evaluation And Discussion}

The previous section claimed that our methodology yields behavior models that can reasonably reproduce individual behaviors. In this section, we investigate the quality of the acquired behavior models through quantitative metrics. First, we evaluate whether the acquired models can well reproduce the transitions in running speed. To do that, we calculated the correlation value between the running speeds of the human subject and those of his/her behavior model. Such a correlation value is a time-tested and an academically accepted index to quantitatively measure the performance of simulations, particularly traffic simulations [20]. Table IV(a) shows correlation values for the running speeds of human subjects $S_{1}, S_{2}$, and $S_{3}$ and their agents. The bold values in the table show the correlation value between human subjects' log data and the corresponding agents' log data. These data confirm that the first two models for
$S_{1}$ and $S_{2}$ reasonably well reproduce the transitions in running speed. Although the correlation value of the model for $S_{3}$ is not as high, it still exceeds 0.60 . The average correlation value for all human subjects was 0.72 . While this is not an outstanding value, we think that the quality of the acquired behavior models is acceptable, given that the behavior models were created using intermingled knowledge. Additionally, from the data shown in this table, we can acquire models that can reproduce individual driving styles. For example, the model for $S_{1}$ is best at reproducing subject $S_{1}$ 's driving style, but it does not well reproduce those of others. The correlation values between $S_{1}$ 's model and $S_{2}\left(S_{3}\right)$ are 0.62 and 0.21 , respectively. In particular, as we can sense from Fig. 4(a), the model for $S_{3}$ is highly uncorrelated. The correlation values for $S_{1}$ and $S_{2}$ are 0.05 and 0.1 , respectively. Accordingly, we have succeeded in acquiring individual driving behavior models, each of which can reproduce the characteristic driving style of a different human subject.

The aforementioned evaluation assessed the agreement in transitions in running speed, but the actual speeds are equally important. Thus, we assessed whether the speeds were similar or not. Fig. 4(b) shows the distribution of running speeds. This figure shows the frequencies of driving at each speed. In this figure, the blue bar is for the human subjects, and the red bar is the result of the behavior models. In Table IV(b), we also plot the average and the standard deviation of the running speed of three examples. We can confirm that there is no crucial disagreement in the standard deviation for all cases, so that the acquired models can well reproduce the driving speeds of the human subjects. In particular, for $S_{1}$, both the transitions in running speed and the actual speeds are well reproduced. Also, for $S_{3}$, both the human subject and his/her behavior model kept the same running speed and the characteristic driving style of using the accelerator frequently. As a result, we can acquire driving behavior models that can reasonably well reproduce individual driving styles of human subjects. While the driving behavior models acquired by our method are reasonable in terms of reproducing individual driving behaviors, they do not achieve accurate behavior modeling. In fact, as shown in Fig. 4(a), there are many gaps between the transitions in running speed 
of the human subjects and acquired models. One main reason for those gaps is the variety of a human's behaviors. A human driver does not always produce the same behavior in an identical situation. This discrepancy can be resolved by employing a probabilistic model, such as a Bayesian network [21], [22]. A Bayesian network allows us to express the dependency between the situation and behavior by assigning probabilities to each potential behavior.

\section{CONCLUSiON}

The agent modeling methodology proposed in this paper represents another direction in agent modeling for realizing humanlike individual agent behavior. Our method does not rely completely on the modeler's knowledge or ability but learns from actual human responses by applying the participatory modeling. We can explicitly obtain information on humans' characteristic behavior, i.e., prior knowledge, through the modeling process and then construct diverse and individual behavior models from the obtained knowledge.

We focused on the traffic domain and encountered several difficulties in constructing agent models due to the lack of prior knowledge. Driving demonstrates many actions whose motivation is hard to explain. If we want a lot of detailed knowledge, we have to spend a lot of time interviewing many human subjects. This represents a bottleneck in knowledge acquisition for modeling. In this paper, we took the approach of using complementary knowledge from other humans in the same situation. As shown in the evaluation conducted here, we could obtain a reasonably well correlated driving behavior from agents. Although we will continue to enhance our methodology, our approach to overcome the lack of knowledge for agent modeling represents a highly attractive first step.

In summary, the contributions of this paper are as follows: 1) to propose a novel agent modeling methodology for realizing individuality in agent behavior; 2) to introduce an approach that can offset knowledge shortfalls for agent modeling; and 3 ) to provide a clue for constructing driver agents for creating realistic traffic simulations.

One future direction of our research is to incorporate other factors that could affect human behavior. For example, emotion plays a crucial role to represent effects within and between humans in the traffic context [23]. We are currently elaborating a model that considers the role of emotions. Another future direction is to simulate traffic flows in urban areas. In order to achieve urban traffic simulations, we need to explicitly model interactions among human drivers.

\section{REFERENCES}

[1] M. Jacyno, S. Bullock, M. Luck, and T. Payne, "Emergent service provisioning and demand estimation through self-organizing agent communities," in Proc. 8th Int. Joint Conf. AAMAS, 2009, pp. 481-488.

[2] L. S. Tesfatsion, "Introduction to the special issue on agent-based computational economics," J. Econ. Dyn. Control, vol. 25, no. 3-4, pp. 281-293, Mar. 2001.

[3] S. M. Lee and A. R. Pritchett, "Predicting interactions between agents in agent-based modeling and simulation of sociotechnical systems," IEEE Trans. Syst., Man, Cybern. A, Syst., Humans, vol. 38, no. 6, pp. 12101220, Nov. 2008.
[4] M. Vasirani and S. Ossowski, "A market-inspired approach to reservationbased urban road traffic management," in Proc. 8th Int. Joint Conf. AAMAS, 2009, pp. 617-624.

[5] T. Moyaux, B. Chaib-draa, and S. D' Amours, "Multi-agent simulation of collaborative strategies in a supply chain," in Proc. 3rd Int. Joint Conf. AAMAS, 2004, pp. 52-59.

[6] L. Panait, "A pheromone-based utility model for collaborative foraging," in Proc. 3rd Int. Joint Conf. AAMAS, 2004, pp. 36-43.

[7] A. Campbell and A. S. Wu, "On the significance of synchroneity in emergent systems," in Proc. 8th Int. Conf. AAMAS, 2009, pp. 449-456.

[8] D. Torii, T. Ishida, and F. Bousquet, "Modeling agents and interactions in agricultural economics," in Proc. 5th Int. Joint Conf. AAMAS, 2006, pp. 81-88.

[9] T. Ishida, Y. Nakajima, Y. Murakami, and H. Nakanishi, "Augmented experiment: Participatory design with multiagent simulation," in Proc. 20th IJCAI, 2007, pp. 1341-1346.

[10] M. Balmer, N. Cetin, K. Nagel, and B. Raney, "Towards truly agent-based traffic and mobility simulations," in Proc. 3rd Int. Joint Conf. AAMAS, 2004, pp. 60-67.

[11] S. Halle and B. Chaib-draa, "A collaborative driving system based on multiagent modelling and simulations," J. Transp. Res. Part C, vol. 13, no. 4, pp. 320-345, Aug. 2005.

[12] P. Paruchuri, A. R. Pullalarevu, and K. Karlapalem, "Multi agent simulation of unorganized traffic," in Proc. 1st Int. Joint Conf. AAMAS, 2002, pp. 176-183.

[13] F. Sempé, M. D. Nguyen, A. Boucher, and A. Drogoul, "An artificial maieutic approach for eliciting experts' knowledge in multi-agent simulations," in Proc. 4th Int. Joint Conf. AAMAS, 2005, pp. 1361-1362.

[14] P. Guyot, A. Drogoul, and S. Honiden, "Power and negotiation: Lessons from agent-based participatory simulations," in Proc. 5th Int. Joint Conf. AAMAS, 2006, pp. 27-33.

[15] T. Conde and D. Thalmann, "Learnable behavioural model for autonomous virtual agents: Low-level learning," in Proc. 5th Int. Joint Conf. AAMAS, 2006, pp. 89-95.

[16] J. M. Vidal and E. H. Durfee, "Predicting the expected behavior of agents that learn about agents: The CLRI framework," Auton. Agents Multi-Agent Syst., vol. 6, no. 1, pp. 77-107, Jan. 2003.

[17] D. Poole, "Theorist: A logical reasoning system for defaults and diagnosis," in The Knowledge Frontier. New York: Springer-Verlag, 1987.

[18] Y. Murakami, Y. Sugimoto, and T. Ishida, "Modeling human behavior for virtual training systems," in Proc. 4th Int. Joint Conf. AAMAS, 2005, pp. $127-132$

[19] R. J. Mooney, "Learning plan schemata from observation: Explanationbased learning for plan recognition," Cogn. Sci., vol. 14, no. 4, pp. 483509, Oct.-Dec. 1990.

[20] J. Hourdakis, P. G. Michalopoulos, and J. Kottommannil, "Practical procedure for calibrating microscopic traffic simulation models," in Proc. TRB 82nd Annu. Meeting, 2003, pp. 130-139.

[21] Y. Xiang and V. Lesser, "On the role of multiply sectioned Bayesian networks to cooperative multiagent systems," IEEE Trans. Syst., Man, Cybern. A, Syst., Humans, vol. 33, no. 4, pp. 489-501, Jul. 2003.

[22] D. Koller and N. Friedman, Probabilistic Graphical Models: Principles and Techniques. Cambridge, MA: MIT Press, 2009.

[23] A. L. C. Bazzan, "Opportunities for multiagent systems and multiagent reinforcement learning in traffic control," Auton. Agents Multi-Agent Syst., vol. 18, no. 3, pp. 342-375, Jun. 2009.

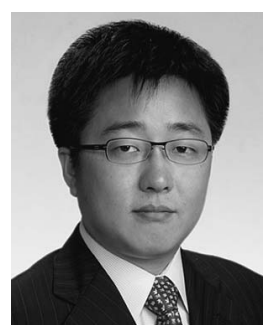

Hiromitsu Hattori received the Ph.D. degree in engineering from the Nagoya Institute of Technology, Nagoya, Japan, in 2004.

He was a Research Fellow of the Japan Society for the Promotion of Science from 2004 to 2007. From 2004 to 2005, he was a Visiting Researcher with the University of Liverpool, Liverpool, U.K. In 2006, he was a Visiting Researcher with the Massachusetts Institute of Technology, Cambridge. He is currently an Assistant Professor with the Department of Social Informatics, Graduate School of Informatics, Kyoto University, Kyoto, Japan. His main research interests include autonomous agents and multiagent systems. He has been working on multiagent-based simulation and human behavior modeling. 


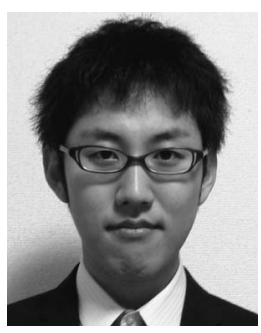

Yuu Nakajima received the Ph.D. degree in informatics from Kyoto University, Kyoto, Japan.

$\mathrm{He}$ is currently an Assistant Professor with the Department of Social Informatics, Graduate School of Informatics, Kyoto University. His research interests include agent modeling, large-scale multiagent systems, and multiagent-based simulations.

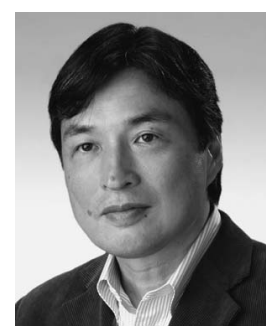

Toru Ishida (F'02) received the Ph.D. degree in engineering from Kyoto University, Kyoto, Japan.

He is currently a Professor with the Department of Social Informatics, Graduate School of Informatics, Kyoto University. Until 1993, he was a Research Scientist with NTT Laboratories. He spent some time at Columbia University, New York, NY; Technische Universitaet Muenchen, Munich, Germany; Universite Pierre et Marie Curie, Paris, France; The University of Maryland, College Park; Shanghai Jiao Tong University, Shanghai, China; and Tsinghua University, Beijing, China, as a Visiting Scholar/Professor. He has been working on autonomous agents and multiagent systems for 20 years now. He also studies social informatics and running research projects related to intercultural collaboration. 